\title{
Comparison of Artificial Neural Network, Fuzzy Logic and Adaptive Neuro-Fuzzy Inference System on Air Pollution Prediction
}

\author{
Reza Amini, S.C. Ng \\ School of Information Technology, SEGi University \\ No.9, Jalan Teknologi, Taman Sains Selangor Kota Damansara, PJU \\ 5, 47810, Petaling Jaya, \\ Selangor, Malaysia
}

reza.amini.b@gmail.com, ashleyng@segi.edu.my

\begin{abstract}
Air pollution can have major impacts on living being and society. Different systems has been developed to predict upcoming air pollution. These prediction systems use different types of models for predicting the air pollution. This paper aims to compare the popular models being used to predict air pollution. The significant models are Artificial Neural Network (ANN), Fuzzy Logic and Adaptive NeuroFuzzy Inference System (ANFIS). These models are not only being applied in air pollution prediction, but they were applied in different fields such as fuel consumption and pattern recognition. The structure of each model are discussed in terms of their advantages and disadvantages. Base on comparison of each model and their structures, Adaptive Neuro-Fuzzy Inference System (ANFIS) is considered to be the best solution for air pollution prediction systems.
\end{abstract}

Keywords: Artificial Neural Network (ANN), Fuzzy Logic, Adaptive Neuro-Fuzzy Inference System (ANFIS), Comparison.

\section{Introduction}

Air pollution has been one of the deliberate problems concerned by the society. It refers to the impurity of the air by harmful chemicals or materials. Air pollution can have effects on health and environments. The short-term effects are throat irritation, headaches and allergic reactions while the long-term effects are kidney damage, lung cancer, heart disease, respiratory disease and brain damage. Besides, 
environmental effects of air pollution includes global climate change, eutrophication and haze (Sierra-Vargas et al., 2012).

Due to these irreparable effects and damages, the need of an air pollution predictive system is crucial. Extensive study and research has been conducted pertaining to this issue and wide range of systems have been developed to solve the problem. Most of the predictive systems that have been developed are using Artificial Neural Network (ANN) (Gershenson, C, 2003). Artificial Neural Network (ANN) has been used for three decades as one of the predictive models and approaches (Baptista, D et al., 2013). Study by Papantoniou and Kolokotsa for prediction of outdoor air temperature has applied Artificial Neural Network (ANN) in four different European cities to collect data from weather stations. Apart from Artificial Neural Network (ANN) other predictive models such as Fuzzy Logic has been used in the same field. Some of these studies are temperature control using Fuzzy Logic (P. Singhala et al., 2014) and application of Fuzzy Logic in transport planning (Sarkar, A., 2012).

\section{Artificial Neural Network (ANN)}

Artificial Neural Networks are relatively electronic models based on the structure of the brain. The brain mainly learns from experience. Artificial Neural Networks modelling also has the potentials of less technical way to develop in machine solutions. The first artificial neural network was designed in 1958 which was called Perceptron (Shanmuganathan, S., \& Samarasinghe, S., 2016). Perceptron proposed how human brain can process visual data and also learn to recognize items. Since then, the number of using neural network was increasing and there have been hundreds of different models developed using artificial neural network. There are differences in these models such as the functions, accepted values, topology, learning algorithms and etc. The learning capabilities and pattern matching of artificial neural network has allowed them to solve many problems that were impossible and difficult to answer by computational or statistical methods (Yadav, N., 2015).

The structure of artificial neural network is inspired by natural neurons. In Figure 1, these neurons take many possible inputs then base on the weight value, it proceeds to the next neuron or produces a 
single output. The neuron needs to be activated in order to proceed to the next neuron or to the output. Activation process of every neuron basically consists of inputs which are multiplied by weight value or the strength of the signal and then computed by a transfer function. These transfer functions must reach a certain threshold. As in Figure 2, Artificial Neural Network can have multiple neurons. Artificial neural network combines the input from every single neuron in each intermediate layer to process information and finally reach a single output (Yadav, N., 2015).
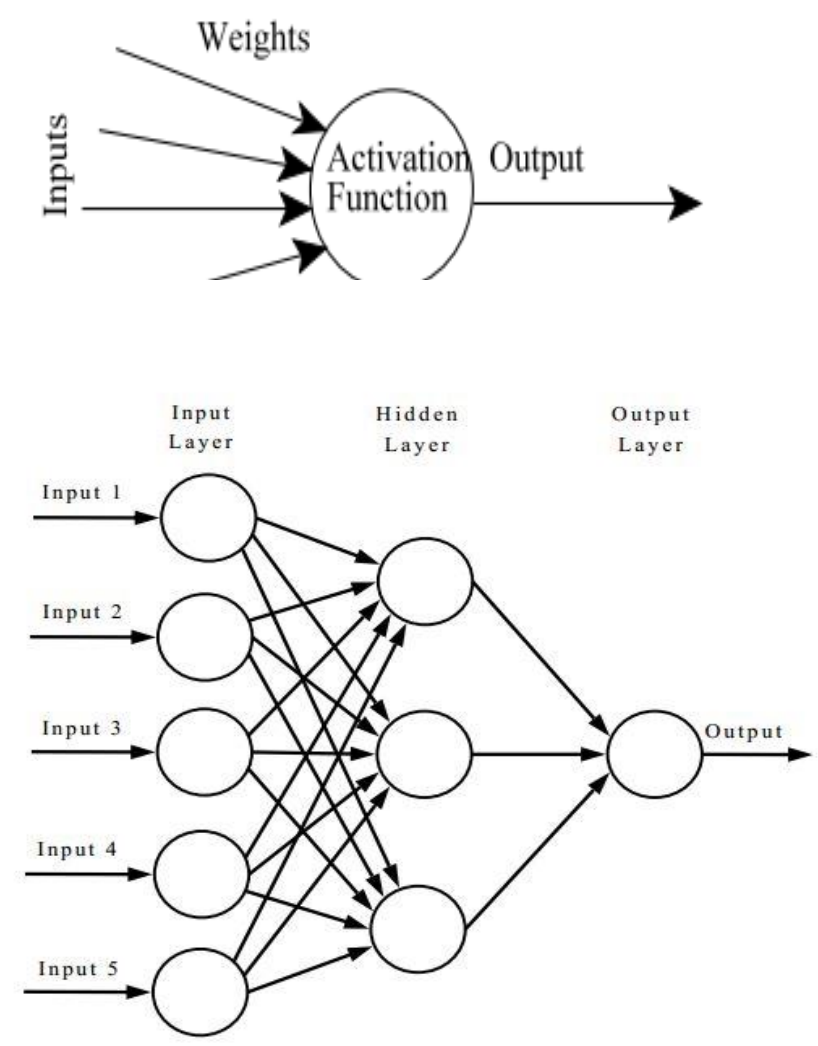

The advantage of artificial neural network is its ability to solve both linear and nonlinear problems. Neural programs is able to learn and it does not require reprogramming the whole system. 
Artificial neural network has disadvantages. It required high processing time for large neural networks. The operation of artificial neural network is highly relying on the training process (Rao, M. A., \& Srinivas, J.,2003).

\section{Fuzzy Logic}

Fuzzy logic is a computation technique used to describe the relationship between the information attributes. Fuzzy logic has a structure that allows that human reasoning capacities to be applied to artificial knowledge base structures. It also offers a high level of computational processing (Wu, Y et al., 2011). As shown in Figure 3, Fuzzy logic has four main processing architectures. These architectures are fuzzification, defuzzification, knowledge base and inference engine. Fuzzification process transforms the crisp set input into fuzzy sets. There is a term called membership function that will work on variables of fuzzy set. Membership function represents a fuzzy sets graphically. Knowledge base is a set of if-then statements that will be provided by the researcher or the domain experts. The inference engine will simulates the human expectations by making fuzzy inference on the input and the if-then rules that previously set in knowledge base architecture. The last architecture which is defuzzification transforms the fuzzy sets which is done by the inference engine into a value.

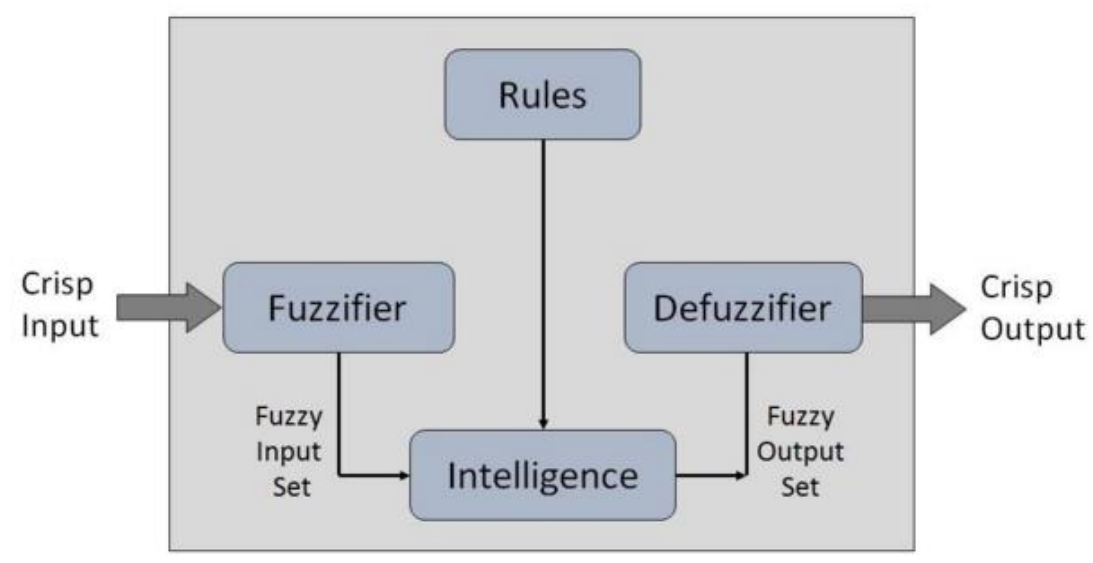

Figure 1 : Fuzzy logic processing architecture 
One of the advantages of fuzzy logic is the simplicity of applying mathematical concept in its fuzzy logics. Due to the flexibility of fuzzy logic modification phase, adding or removing rules are simpler. On the other hand, fuzzy logic has number of disadvantages. It still requires expertise to understand and develop a fuzzy system and it is time consuming to develop fuzzy rules and membership functions (Shamim, M et al., 2011).

\section{Adaptive Neuro-Fuzzy Inference System (ANFIS)}

Adaptive Neuro-Fuzzy Inference System is an adaptive network which make use of both artificial neural network and fuzzy logic. By combining both of the methods, this network not only will inherit the benefits and characteristic of them but also will eliminate their lonely usage. The operation of Adaptive Neuro-Fuzzy Inference System is feed forward which means that the information starts from the first neuron or node and is carried forward to the next node until the output result is achieved. Adaptive Neuro-Fuzzy Inference System consist of two learning method: hybrid and back propagation learning methods. Below is the typical architecture of Adaptive Neuro-Fuzzy Inference System which the circles indicates fixed node and square indicates adaptive nodes (Taher, A. 2010).

Base on Figure 4, the first and last layer of the network are considered as input and output nodes. The hidden layers consist of membership function nodes. This structure assembles the normal feed forward network which is crucial for the viewer to modify or realize. The function of each layer is described as follows (Taher, A. 2010):

Input layer (Layer 1): In this layer every node consist of membership functions. Any parameter that passes to these nodes will be considered as premise or foundation parameters.

Rule nodes (Layer 2): As shown in Figure 4, all nodes in this layer are represented as circle type of nodes. The outcome of these nodes depends on the strength of the rule. Operators such as AND or are used to get the output results.

Average nodes (Layer 3): This is a circle type of node with the labelled as N (Figure 4). Every node in this layer calculate the weight 
of each input. Outputs from these nodes are called normalized firing strength.

Consequent nodes (Layer 4): This layer consists of transfer functions. The rules toward the output or the functions defined is calculated in this layer.

Output node (Layer 5): This layer is the last step of ANFIS and it calculates all overall result and output by calculating the incoming signals.

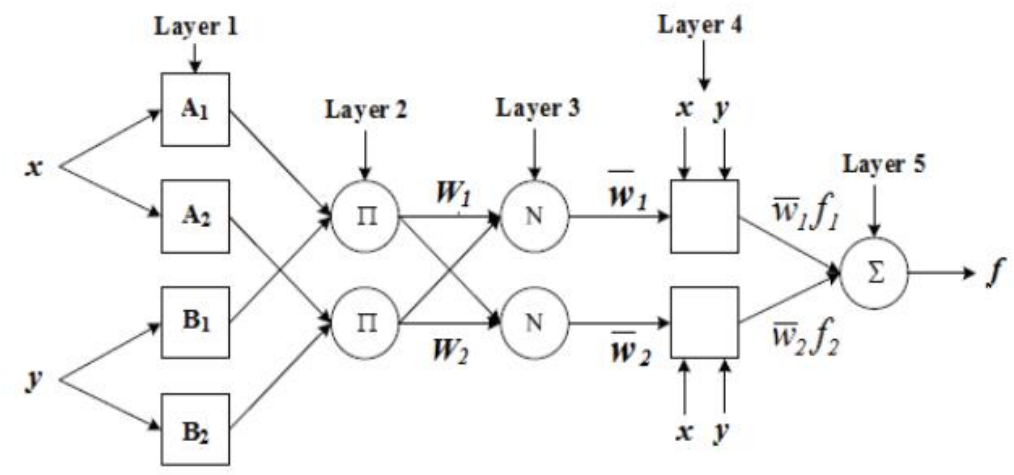

Figure 2 : ANFIS architecture

The advantages of Adaptive Neuro-Fuzzy Inference System can be refer to its fast and accurate learning, easy implementation, easy to incorporate in both linguistic and numeric knowledge for problem solving (Azar, A. T., 2014). Adaptive Neuro-Fuzzy Inference System is capable of solving complex nonlinear problem since it uses both artificial neural network and fuzzy logic (José Kleiton Ewerton Da Costa Martins, \& Araújo, F. M. 2015). The disadvantage of Adaptive Neuro-Fuzzy Inference System is its augmented decline in performance compared to other methods such as artificial neural network when the system has lack of data for its training purpose.

In this study, a case study has been conducted to compare the performance of these three methods. A study by Atmaca et al. (2001) shows the predictions of fuel consumption of automobiles by applying Adaptive Neuro-Fuzzy Inference System, Artificial Neural Network and Fuzzy Inference Systems. This study compared the outcomes of fuel consumption prediction conducted by each method. The data for 
the comparison were obtained from University of California at Irvine. The data collected have been studied under the same condition to the three methods. This comparison shows the performance of each method and the data simulations were performed using MATLAB. As the comparison of the result, it shows that ANFIS hit its expected output target faster than neural network. It means that ANFIS learning duration is shorter in comparison to neural networks. In case of huge data or complicated systems the use of ANFIS would be more useful compare to the other two methods. In training procedure of ANFIS outcomes are with minimum total error. This means that the best learning method between the three methods is ANFIS. However total error of neural network is smaller than ANFIS when the trained variables were tested against checking data but this could happen because of less number of data for its training purpose. Between the three methods Fuzzy logic method has the least promising outcome. The limitations of rule size and membership variable restriction are the reasons of getting such result.

\section{Conclusion}

As a conclusion of comparing the three methods, ANFIS is considered a suitable method for prediction of air pollution comparing to neural network and fuzzy logic methods as lonely usage. Air pollution prediction involves high dimensional multivariate data analysis. The prediction process is highly nonlinear and dynamic. By combining the advantages of both neural network and fuzzy logic methods, ANFIS is expected to produce more accurate results in shorter time.

\section{References}

Sierra-Vargas, M. P., \& Teran, L. M. (2012). Air pollution: Impact and prevention. Respirology, 17(7), 1031-1038.

Gershenson, C. (2003.). Artificial Neural Networks for Beginners. Artificial Neural Networks for Beginners, 229-231.

Papantoniou, S., \& Kolokotsa, D. (2016). Prediction of outdoor air temperature using neural networks: Application in 4 European cities. Energy and Buildings, 114, 72-79. doi:10.1016/j.enbuild.2015.06.054 
P. Singhala., D. N. Shah., \& B. Patel. (2014). Temperature Control using Fuzzy Logic. IJICS International Journal of Instrumentation and Control Systems, 4(1), 1-10. doi:10.5121/ijics.2014.4101

Shanmuganathan, S., \& Samarasinghe, S. (2016). Artificial neural network modelling.

Yadav, N. (2015). An introduction to neural network methods for differential equations. Dordrecht: Springer.

Rao, M. A., \& Srinivas, J. (2003). Neural networks: Algorithms and applications. Pangbourne, Eng.: Alpha Science International.

Sarkar, A. (2012). Application of Fuzzy Logic in Transport Planning. IJSC International Journal on Soft Computing, 3(2), 1-21. doi:10.5121/ijsc.2012.3201

Wu, Y., Zhang, B., \& Lu, J. (2011). Fuzzy Logic and Neuro-fuzzy Systems: A Systematic Introduction.

Shamim, M., Enam, S., Qidwai, U., \& Godil, S. (2011). Fuzzy logic: A "simple" solution for complexities in neurosciences? Surgical Neurology International Surg Neurol Int, 2(1), 24. doi:10.4103/2152-7806.77177

Taher, A. (2010). Adaptive Neuro-Fuzzy Systems. Fuzzy Systems, 86-109.

Atmaca, H., Cetişli, B., \& Yavuz, H. (2001). The Comparison of Fuzzy Inference Systems and Neural Network Approaches with ANFIS Method for Fuel Consumption Data. In Proceedings of the 2nd International Conference on Electrical and Electronics Engineering Papers ELECO'2001, Bursa, Turkey, 2001. 
Azar, A. T. (2014). Neuro-fuzzy feature selection approach based on linguistic hedges for medical diagnosis. IJMIC International Journal of Modelling, Identification and Control, 22(3), 195. doi:10.1504/ijmic.2014.065338

José Kleiton Ewerton Da Costa Martins, \& Araújo, F. M. (2015).

Nonlinear System Identification based on Modified ANFIS. Proceedings of the 12th International Conference on Informatics in Control, Automation and Robotics. doi:10.5220/0005544905880595 